Shakespeare et le cinéma

\title{
Richard III de Raoul Ruiz : entre difformités et déformations
}

Laetitia Coussement

\section{(2) OpenEdition}

Journals

\section{Édition électronique}

URL : http://journals.openedition.org/shakespeare/210

DOI : 10.4000/shakespeare. 210

ISSN : 2271-6424

Éditeur

Société Française Shakespeare

Édition imprimée

Date de publication : 1 novembre 1998

Pagination : 77-90

ISBN : 2-84269-230-6

\section{Référence électronique}

Laetitia Coussement, "Richard III de Raoul Ruiz : entre difformités et déformations 》, Actes des congrès de la Société française Shakespeare [En ligne], 16 | 1998, mis en ligne le 01 novembre 2007, consulté le 19 avril 2019. URL : http://journals.openedition.org/shakespeare/210 ; DOI : 10.4000/

shakespeare. 210 


\section{S H A K E S P E A R E \\ \& $\quad$ L E $\quad$ C I N É M A}

Société Française Shakespeare

Actes du Congrès de 1998

米 $*$ *

Textes réunis et présentés par

Patricia DORVAL

publiés sous la direction de

Jean-Marie MAGUIN 
Site web : <http : //alor.univ-montp3.fr/serinf/SFS/> Liste de diffusion : <sfs-1@smrl.univ-montp3.fr >

Tous droits de traduction, de reproduction et d'adaptation réservés pour tous les pays.

(C) 1998. Société Française Shakespeare,

École Normale Supérieure, 45 rue d'Ulm. 75005 Paris.

ISBN 2-84269-230-6 


\section{R ICHARD III DE RAOUL RUIZ: ENTRE D IF FOR M ITÉS E T DÉ F O R M A T O N S}

Richard III de William Shakespeare a fait l'objet de nombreuses adaptations cinématographiques, des films muets du début du siècle - trois en 1908, 1911 et 1912 - au récent Looking for Richard, d'Al Pacino (1996) en passant par le Richard III de Laurence Olivier en 1956 et celui de Richard Loncraine en 1995. A ces versions anglaises et nord-américaines, il convient d'ajouter celle du cinéaste chilien Raoul Ruiz. Réalisateur prolifique el protéiforme, écrivain, théoricien de cinéma, metteur en scène de théâtre, conteur, comédicn à l'occasion ${ }^{1}$, ce «bricoleur de génie» ${ }^{2}$ a une filmographic impressionnante. Dans un entretien, il déclare : «On m’a reproché en gros deux choses: primo, de faire trop de films, mais je n'y peux rien, c'est dans ma nature; secundo, d'aller trop vers l'obscur" ${ }^{3}$. Je ne citerai ici que quelques titres en guise de points de repères: Généalogies d'un crime (1997), Trois vies et une seule Mort (1996), Fado, majeur et mineur (1994), L'île au trésor (1986), L'éveillé du pont de l'Alma (1985), Les trois couronnes du matelot (1982), L'hypothèse du tableau volé (1978). C'est en 1986 que Raoul Ruiz réalise un Richard III méconnu. Le film fut projeté en avant-première à Cannes mais ne fut jamais diffusé.

Ce Richard III est issu d'une triple rencontre: William Shakespeare, Georges Lavaudant et Raoul Ruiz. En 1984, G. Lavaudant - co-directeur de la maison de la culture de Grenoble - met en scène Richard III pour la cour d'honneur du Palais des Papes, spectacle qui connaît un grand succès. La maison de la culture de Grenoble encourage à cette époque les «projets mixtes» qui associent théâtre, danse, cinéma et musique. C'est dans ce 
contexte que Georges Lavaudant donne sa pièce au cinéaste Raoul Ruiz en lui disant : "Fais-en ce que tu veux». Raoul Ruiz crée une cuvre à part entière car il ne se contente pas de filmer la mise en scène de G. Lavaudant - il ne s'agit pas d'unc captation. Il recrée sa propre mise en espace cinćmatographique avec décors (naturels et artificiels), éclairages, déplacements, figurants, et de nombreux ajouts personnels. Pour finir, il n'cmprunte que les comédiens et les costumes. Le texte reprend la traduction dc J.-M. Déprats déjà utilisée par G. Lavaudant ${ }^{4}$. De G. Lavaudant il ne subsiste qu'une apparition fugitive puiqu'on l'aperçoit métamorphosé dans le rôle de la duchesse d'York ${ }^{5}$. Or c'est bien de métamorphoses qu'il est question dans ce film : outre Georges Lavaudant, Philippe Morier-Genoud, au visage déformé par des tics, tient le rôle de Margaret, Marc Betton campe un Édouard IV puis un Catesby méconnaissables, sans oublier le maître incontesté des transformations, Ariel Garcia-Valdès qui incarne un Richard grimaçant.

L.e thème de la déformation constitue un des fils directeurs du film. A l'origine se trouve Richard, individu contrefait, «Difforme, inachevé, dépêché avant terme / Dans ce monde haletant à peine à moitié fait...» (I.1), s'employant en retour à contrefaire le monde. S'inspirant de Richard, R. Ruiz met in ceuvre une esthétique de la déformation, revendiquant tous les effets du septic̀me art : trucages, usage de filtres, changements de focales, jeux d'ombre et de lumièrc, faux raccords, mouvements de caméra complexes.

$\mathrm{Cc}$ jeu permanent sur la forme conduit l'auteur et le spectateur à s'interroger sur la nature même du cinéma: art de la reproduction ou de la déformation?

Le premic̀re déformation opérée par Raoul Ruiz est loin d'être négligeable. Certains parleraient même de mutilation: il ne traite que les deux tiers de la pièce et interrompt le film au couronnement de Richard et à l'assassinat des enfants d'Édouard. Questionné à ce sujet, Raoul Ruiz répond que scule l'ascension de Richard l'intéresse. Pour résumer son film, il déclare avec une pointe de provocation : «Ce sont des nains qui parlent français» 6 .

Lc tout premier plan révèle un ciel parcouru de nuages ainsi qu'une inscription au bas de l'image : «Mildendo... 203 avant Gulliver». Ainsi Raoul Ruiz place d'emblée la pièce de Shakespeare sous l'égide de l'univers swifticn et plus particulièrement des lilliputiens auquels lui-même, à la suite de G. Lavaudant, comparait les comédiens. C'est une famille royale de monstres sortis d'un tableau de Vélasquez qu'il nous donne à voir. R. Ruiz reprend l'appareillage déjà utilisé dans la mise en scène de G. Lavaudant, une prothèse qui empêche Ariel Garcia-Valdès de se mouvoir normalement et lui 
confère cette démarche instable et erratique. Mais il étend les déformations ct les difformités à d'autres personnages : on pense à Catesby - Marc Betton et son ceil de verre - et plus généralement à tous les comédiens dont les visages, fardés à l'extrême, évoqueraient des masques.

Dans la pièce de Shakespeare, l'intérêt de Richard ne réside pas tant dans sa difformité que dans sa propre capacité déformante. Sa propension à se déformer au gré des circonstances et à déformer le monde font de lui un monstre. Héritier du Vice des moralités, aussi ambivalent qu'Ambidexter dans Cambises de Thomas Preston (1561), il est passé maître dans l'art de la duplicité comme le rappelle la duchesse d'York:

Ah, se peut-il que la Duplicité escroque une figure si douce,

Et sous un masque vertueux cache un Vice profond! (11.2)

Mais on ne saurait réduire Richard à n'être qu'un avatar du Vice ; il incarne simultanément plusieurs rôles ${ }^{7}$ : le tyran, l'antéchrist, le machiavel, le fléau de Dieu. Surtout, il est l'un des tous premiers personnage / acteur / metteur en scène du répertoire shakespearien, préfigurant Falstaff, Hamlet, Rosalind, Iago ou Prospero. Richard joue et se regarde jouer avec la complicité de son cousin, Buckingham, lui aussi comédien :

\section{Voyons, cousin, sais-tu frissonner et changer de coulcur. \\ Etouffer un souffle au milieu d'un mot, \\ Et puis le reprendre, et t'arrêter encore, \\ Comme si tu étais égaré et fou de terreur? (III.5)}

Tout le côté histrionique de Richard est magistralement servi dans le film par l'interprétation versatile et ludique d'Ariel Garcia-Valdès. Dès le premier monologue et durant toute la pièce, Richard apparaît comme un personnage double, scindé, ainsi que le suggèrent les références constantes à son ombre: "Je n'ai d'autre plaisir pour passer le temps, / Que d'épier mon ombre au soleil... » (I.1). Renversant la perspective traditionnelle, Richard ne s'intéresse pas au soleil en tant que tel mais à l'ombre qu'il projette sur lui : «Resplendis, beau soleil, en attendant que j'achète un miroir, / Que je puisse en marchant mon ombre apercevoir» (I.2). Le mot «shadow» mérite que l'on s'y attarde : l'un de ses multiples emplois, attesté à l'époque élisabéthaine ${ }^{8}$, renverrait au théâtre et à l'acteur. Dans le contexte de la pièce, l'usage récurrent de l'ombre, «shadow», souligne à la fois l'importance du jeu de l'acteur et celle accordée aux apparences, aux simulacres, aux dépens de la substance. Ironiquement, c'est par les ombres que Richard périra à la bataille de Bosworth : «les ombres de cette nuit / Ont jeté plus de terreur dans l'âme de Richard / Que la substance de dix mille soldats...» (V.3). Ironie encore lorsque la duplicité dont Richard était jusque-là le maître incontesté se 
retourne contre lui en la personne de Stanley qui lui avait juré fidélité, «Je n’ai jamais été, et ne serai jamais, un traître» (IV.4), et qui le trompera à la bataille de Bosworth.

Richard construit son pouvoir sur la déformation des faits et le mensonge. Il manipule les mots au même titre que les apparences, le language ćtant son arme de prédilection. Dès la première scène, il joue sur l'ambiguité et l'ćlasticité de la lettre «G», G de Georges ou de Gloucester ?

Ainsi, comme Iniquité, le Vice des moralités, J'interprète deux sens en un mot. (III.1)

Dans la scène 5 de l'acte III, il ordonne à Buckingham d'insinuer auprès du maire el des citoyens la bâtardise des enfants d'Édouard et d'Édouard luimême, afin de légitimer sa prétention au trône. Faisant exploser la rhétorique de ses interlocuteurs - en jouant sur la stichomythie par exemple - il retourne les mots contre ses adversaires, comme le montre la scène du duel verbal avec Lady Anne (1.2). De nombreux critiques ont mis l'accent sur limportance de la rhétorique dans cette pièce de jeunesse - on pense en priorité à la scène de lamentation sénéquéenne des reines - rhétorique qui conduit à un certain formalisme comme le souligne A. Hammond :

This formality has the effect of marmorealizing the scenes into a 'wall of stone music', a rigid formalized kind of structure into which Richard's protean mutability strikes with the force of a mason's hammer.

Or R. Ruiz met lui aussi l'accent sur l'importance du rituel, sur la dimension formelle de la pièce, usant de la profondeur de champ pour souligner la théâtralité. Il va même jusqu'à rendre littéralement visible «l'effet marmoréen» dont parlait A. Hammond, en insérant à plusieurs reprises un personnage mystéricux qui sculpte au burin des bustes en marbre. Si le cinćaste a perçu le formalisme de la pièce shakespearienne et a su en tirer parti, il exploite aussi l'autre dimension de l'œuvre, l'aspect protéiforme, la mutabilité, incarnés principalement par Richard de Gloucester. Sa caméra s'apparenterait alors à un miroir anti-réaliste, voire à un miroir déformant, ou cncore à un miroir brisé qui fait éclater le réel en fragments disparates.

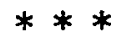

La première de ces disparités concerne le décalage entre la voix et l'image. Dès les premiers plans, les paroles de Richard sont décalées par rapport à ce que l'on voit à l'écran. Tandis qu'il entame son monologue, 
"Ores voici l'hiver de notre déplaisir / Changé en glorieux été par ce soleil d'York ; / Et tous les nuages qui menaçaient notre Maison / Ensevelis au sein profond de l'océan», apparaît à l'écran un ciel nuageux, traversé de fumées noires, et dans le plan suivant une main brandissant une épée. Le décalage est ici poussé jusqu'à la contradiction, créant un effet d'étrangeté recherché par Raoul Ruiz: "Ce qui m'intéresse ce sont plutôt toutes les possibilités de relations, de malentendus, entre ce qu'on voit et ce qu'on dit» ${ }^{10}$.

Cette voix hors champ récitant le premier monologue semble surgir de nulle part, et pour un spectateur qui ne connaît pas la pièce, elle n'est pas immédiatement identiliable. L'espace d'une seconde, il pourrait s'agir d'une «voix off». Mais le plan séquence qui suit, composé d'un panoramique vertical puis horizontal et d'un plan d'ensemble, nous révèle progressivement celui qui parle et l'endroit où il se trouve. Au gros plan sur le visage de Richard étendu sur le sol parlant à la caméra, succède un plan d'ensemble sur un paysage désolé, champ de bataille jonché d'armures vides, vestiges de la guerre à peine achevée. $R$. Ruiz réutilise le même procédé, jouaint sur l'ambiguïté d'une «voix off» qui en réalité n'en est pas une, lors de la première apparition de Lady Anne. Tandis qu'à l'arrière-plan, on aperçoit des personnages en deuil, au premier plan des silhouettes vêtues de noir, poussant des landaus, traversent le champ. Simultanément s'élève une voix de lemme impossible à localiser. La caméra fait alors un gros plan sur le visage voilé de Lady Anne, ce qui permet au spectateur d'identifier enfin le locuteur. Ces effets de disparité sont renforcés par la présence «d'artéfacts», la multiplication de signes qui encombrent l'écran et saturent l'image afin de nous éloigner de l'action principale.

Tout au long du film, le spectateur est intrigué par la présence de détails insolites dans le champ. La première scène de taverne débute par un plan aussi surprenant qu'énigmatique : à l'arrière-plan se trouve Richard dont on entend le rire avant qu'il ne parle, tandis qu'au premier plan, une main verse un liquide dans une choppe dont semble s'échapper un poisson. L'attention du spectateur est inévitablement détournée vers ce détail aux dépens de la tirade de Richard. Peu après, Richard est filmé en contre-plongée, assis à une table couverte de cadavres de bouteilles en amorce qui le dissimulent en partic. Le cinéaste joue souvent sur l'effet de surprise que peuvent produire certains gros plans, comme dans cette image où apparaît le visage de profil d'un noble renversé en haut de l'image tandis qu'à l'arrière-plan on distingue Édouard et Élisabeth dans une grotte. Dans le même esprit, il fait aussi des gros plans sur la main d'un personnage : le plan sur le dé à coudre posé sur le doigt de Richard reste sans doute le plus mystérieux.

Cet usage répété d'objets en amorce pose un problème de pondération au sein de l'image : quelle attention faut-il accorder à quel objet et pourquoi ? 
Fréquemment, le premier plan en amorce et le dernier plan en profondeur de champ ne semblent pas entretenir de relation logique ou dramatique évidente, ce qui parasite et brouille la compréhension du spectateur. Cette surcharge de l'image par des objets saugrenus fait partie de l'humour ruizien; elle est aussi l'indice d'un espace cinématographique saturé, constitué de profondeurs multiples, de prolongements, de rappels, d'échos.

La première rencontre entre Richard et Lady Anne témoigne de cette volonté de remplissage maximal du cadre. Le cinéaste aurait pu se contenter de filmer les deux protagonistes en champ / contrechamp - ce que fait Richard L.oncrainc dans son Richard III. Dans le cas présent, on assiste à une véritable mise en scène grâce à l'utilisation de la profondeur de champ. Au fond se trouvent Lady Anne et le cortège tandis qu'au premier plan se découpe la main de Richard, indice métonymique de sa présence. R. Ruiz inclut dans le champ les figurants du cortège qui deviennent alors eux-même spectateurs de la scène et ponctuent les répliques des deux protagonistes de sauts, de claquements de doigts, de coups frappés, voire d'applaudissements. Plus qu'à une «mise en ahyme», par ailleurs entièrement cohérente avec l'aspect délibérément formel, rhétorique et théâtral de la scène, c'est à un rituel parodique et grotesque que nous convic ce choreur délirant.

R. Ruiz crée aussi des effets de décalage en exploitant les possibilités de changements de focales. Ainsi la scène où apparaît $G$. Lavaudant déguisé en duchesse d'York est construite sur des changements de mise au point et une bascule des plans : dans un premier temps, le premier plan contenant le visage d'Élisabeth est vu nettement tandis que dans un arrière-plan flou, la silhouctte de la duchesse se dessine derric̀re un voile transparent; dans un second temps, le visage d'Élisabeth devient flou et la mise au point se fait sur le visage de profil de la duchesse. Un jeu avec le diaphragme qui s'ouvre et se ferme vient compléter ces effets d'apparition et de disparition. La caméra serait ici assimilable à un oil défectueux, contraint d'effectuer une mise au point, d'accommoder, afin de voir nettement les différents plans d'un univers déformé.

La musique du film, composée par Jorge Arriagada, joue elle aussi un rôle essenticl dans ces effets de disparité. Là encore, le décalage est constant entre une musique grandiloquente, épique, et des personnages dérisoires assimilés à des nains ou des petits monstres. De ce déséquilibre naît un humour bouffon, déjà présent dans la mise en scène de G. Lavaudant accompagnée de mandolines. La scène où apparaît Lady Anne escortant la dépouille d'Henry VI est exemplaire. Alors que le cortège funèbre se rapproche, les musiciens qui le composent se mettent à jouer de plus en plus faux. La musique solennelle du deuil est remplacée par une cacophonie de flûtes accompagnée des grognements des porcelets portés par certains figurants. 
Ces décalages, outre le jeu, la surprise et l'humour qui en découlent ingrédients indispensables des films de R. Ruiz - seraient à rapprocher d'une stratégie de la disproportion chère au cinéaste.

Il est remarquable que R. Ruiz n'utilise quasiment jamais le point de vue dit «naturel». En revanche il use et abuse de plongées et de contre-plongćes qui donnent au spectateur une vision déséquilibrée du monde, voire une vision d'un monde en déséquilibre. L'exemple le plus frappant concerne Richard. La versatilité de l'acteur n'a d'égal que la mobilité de la caméra qui le suit. Dans les tous premiers plans se succèdent les contre-plongées, les gros plans sur le visage de Richard puis sur la tête du cheval, le tout avec une caméra portée qui accentue l'effet bougé. Un faux raccord - le cheval est d'abord filmé de droite à gauche puis de gauche à droite - vient renforcer l'effet de déstabilisation du spectateur. Les mouvements de caméra et la multiplication des points de vue empêchent une vision unifiante de la réalité et fragmentent le monde jusqu'au vertige.

De plus, R. Ruiz n'hésite pas à exagérer certains détails. Ainsi les dernières images du film mettent l'accent sur le meurtre des enfants d'Édouard au moyen d'un gros plan sur les mains de Tyrrel, mains monstrueuses qui semblent contenir Richard et son cheval. Eisenstein soulignait la perversité de la perspective au cinéma : «les lois de la perspective cinématographique sont telles qu'un cafard en gros plan paraît plus redoutable que cent éléphants pris en plan d'ensemble» "I. Or R. Ruiz se complaît à cette subversion des perspectives et des proportions traditionnelles. Ce jeu sur la disproportion ne fait que traduire les multiples indices de déséquilibre déjà contenus dans la pièce de Shakespeare. On se souvient du dialogue entre Hastings et Catesby sur la mauvaise santé de l'État:

Hast. Quoi de neuf, quoi de neuf dans notre État qui titube?

Cat. En vérité, c'est un monde bien chancelant, mon seigneur,

Et je crois qu'il ne tiendra jamais droit,

Tant que Richard ne portera pas la guirlande du royaume.

Le cinéaste propose une interprétation à la fois originale et pertinente de cette scène; il nous montre Hastings, un bandeau sur les yeux, se guidant maladroitement d'une canne, cherchant son chemin dans un labyrinthe de toiles peintes. La cécité momentanée de Hastings ainsi que sa démarche hésitante, l'œil de verre de Catesby, transposent la perte des repères, les apparences trompeuses, le doute, que Shakespeare met en scène dans sa pièce 
par l'intermédiaire de l'effondrement du pouvoir, du royaume et du monde. Autant de remises en cause du regard et donc de la perception du réel chez le cinéaste Ruiz, qui font écho à la vision shakespearienne d'un monde en désintégration. Comme le rappelle Serge Toubiana, la filmographie de R. Ruiz est parcourue de personnages qui voient mal :

Tout le cinéma de Ruiz d'ailleurs est tordu, vu à travers de curieux prismes, et dénature la perspective classique : un cinéma de «borgne» (le titre d'un de ses récents films). ${ }^{12}$

Au scin de cet univers déformé, il n'est guère surprenant que le texte soit lui aussi soumis à des transformations. R. Ruiz est réputé pour ses dysnarrations, ses narrations parallèles, entrecoupées, fragmentées, ses enchevêtrements complexes qui visent à égarer le spectatcur dans des filmslabyrinthes. Le Richard $I I I$ ne fait pas exception. Les vingt premières minutes ne reproduisent pas fidèlement l'enchaînement des scènes de la pièce ; le monologue initial de Richard est scindé en deux parties : les treize premiers vers de Richard, puis l'arrivée de Clarence prisonnier, suivie du monologue de Lidy Anne et de la fin du monologue de Richard. Le jeu de découpage et de collage avec le texte shakespearien contribue à la structure disparate du film. Le spectatcur se trouve face à une succession de tableaux dont le lien logique reste parfois difficile à établir. Ce Richard III est très peu explicatif contrairement à la limpidité du Looking for Richard d'Al Pacino, film pédagogique qui explicite pour le profane les tenants et les aboutissants de la guerre des Deux Roses, ou à la transposition réaliste de Richard Loncraine qui silue l'action dans une Angleterre fasciste en référence à Oswald Mosley ct aux années trente. L'œuvre de R. Ruiz est constituée de manques que le spectatcur tente de combler, comme les paroles ambiguës de Richard qu'il faut interpréter. Ce Richard III témoignerait de cette volonté d'inachèvement, d'incomplélude chère au cinéaste :

Parce qu'un film est de toute façon un objet inachevé, $[\ldots]$. Il faut donc le considérer comme un parc anglais où il y a des ruines auxquelles on a intégré des choses et qu'on utilise de façon différente. Le parc anglais est construit autour du château pour s'égarer. Le plaisir consiste à s'égarer et non à rentrer dans le château pour se défendre contre les barbares. ${ }^{13}$

R. Ruiz nous offre un Richard III expérimental, fantastique, onirique, dont l'ultime déformation serait le recours constant à l'artifice. 
Dans son article, «Le vrai, le faux, le factice» ${ }^{14}$, Alain Bergala rapproche $\mathrm{O}$. Welles et $\mathrm{R}$. Ruiz pour leur jeu avec les puissances du faux. $\mathrm{R}$. Ruiz a effectivement recours à de nombreux artifices, qu'il s'agisse du décor, des éclairages, des trucages, de la théâtralité, ou encore des citations cinématographiques et picturales qui émaillent le film.

Le film fut réalisé au Monteynard près de Grenoble, sur la route Napoléon. La plupart des scènes furent tournées en plein air, dans le cadre désolé entourant le lac glaciaire. Au lieu peu avenant vint s'ajouter la combustion permanente de pneus dégageant cette fuméc noire si caractéristique. Les quelques scènes d'intérieur, celles de la taverne et de la prison, furent tournćes dans la forteresse de la Bastille à Grenoble. Enfin, un décor kitsch de grotte artificielle en carton-pâte tenant lieu de palais, fut aménagé dans la salle du Cargo à Grenoble. Il est impossible de situer l'action dans un lieu ou à une époque précise. Seuls les costumes, dont la richesse contraste avec la désolation du lieu, empruntés à la mise en scène de G. Lavaudant en référence à la période élisabéthaine fournissent un ancrage temporel dans ce «no man's land». Le premier plan suggère une date aussi lointaine qu'incertaine, «203 avant Gulliver». Le cinéaste situe donc son Richard III dans un monde de légende, monde maléfique, chaotique et inachevé, comme Richard. L'eau y occupe une place prépondérante: la présence du lac dès les premières scènes, l'arrivée de Clarence en barque, le bruit constant de l'eau qui ruisselle dans la grotte, le visage d'Édouard IV mort dans la vasque, les reflets aquatiques, sont autant de signes qui évoquent un monde instable, fuyant, comme ces poissons que des figurants tentent d'attraper au bord du lac. Pascal Bonitzer qualifie la version ruizienne «d'approche quasi-biologique, endoscopique, de l'univers shakespearien» ${ }^{15}$ : «Tout scintille, tout bave, tout flotte dans une lumière translucide, dans une atmosphère de grotte marine, de membrane placentaire...» ${ }^{16}$. L'univers ruizien est marqué par une régression vers un monde primitif où les quatre éléments sont omniprésents. C'est leur affrontement qui intéresse le cinćaste, comme dans ces gros plans sur une eau terreuse bouillonnante ou encore dans cette image complexe où les flammes des bougies s'opposent aux gouttes d'eau tombant des stalactites. S'agit-il d'un monde en gestation ou au contraire en dissolution comme semble l'indiquer cette ruine peinte sur la toile qui apparaît à plusieurs reprises?

Venant compléter le décor, les éclairages jouent un rôle capital dans la création d'une ambiance surnaturelle. A cet effet, $R$. Ruiz a recours à des lumières rasantes, mais aussi à des bougies, ou encore à des filtres. Un plan en particulier montre Richard avançant de sa démarche chaotique vers la 
caméra. Le sol composé de cailloux blancs est vivement éclairé par des projectcurs ce qui produit un effet de fluorescence, comme si la lumière provenait du sol même. Le contraste entre l'épaisse nuit environnante, la silhouctte noire de Richard et la blancheur surnaturelle du sol est saisissant, contraste renforcé par un maquillage qui lui donne l'aspect d'un vampire échappé d'un film expressionniste. Le même procédé est utilisé pour le dernier plan du banquet de réconciliation qui s'achève dans la nuit complète tandis que l'éclairage de la nappe blanche produit cet effet fluorescent. La table du festin semble alors flotter en apesanteur dans un espace indistinct où sol et ciel, haut et bas se confondent. R. Ruiz joue aussi des possibilités de lumière poudreuse, de saturation lumineuse, particulièrement dans la grotte, lorsque Richard rencontre les deux jeunes princes. Tous les effets de diffusion de la lumière, effets parasites, reflets, sont non seulement visibles mais appuyés, entourant les personnages d'un halo surnaturel. Signalons enfin l'utilisation de filtres de couleur : la première moitié du banquet est filmée sur un fond de cicl incandescent grâce à l'utilisation d'un filtre rouge-orangé, tandis que la seconde est lilmée de nuit.

Poussant à l'cxtrême un parti-pris d'irréalisme, le cinéaste fait aussi appel à de nombreux trucages. A plusieurs reprises, il a recours à des procédés de surimpression comme pour l'arrivée des deux exécutcurs de Clarence dans le dos de Richard, ce qui leur confère une dimension spectrale. De même, lorsque Clarence s'endort, les deux meurtriers qui pénètrent dans la prison semblent émerger des feuilles mortes qui recouvrent le visage du duc. Le cinćaste utilise un autre procédé remarquable, un fondu enchaîné spatialisé, lorsque les visages de Buckingham et de Richard se statufient successivement de gauche à droite et non simultanément. Outre «l'effet marmoréen" déjà mentionné, cet artifice peut aussi être interprété comme un clin d'œil humoristique aux visages des présidents américains de Mount Rushmore.

R. Ruiz s'affirme donc comme le maître d'un art visuel des effets, art qui trompe et détrompe en même temps, qui oscille entre l'artifice et la dénonciation de cet artifice. Il souhaitait conserver le jeu théâtral des acteurs de la mise en scène de G. Lavaudant et accentua la théâtralité du film, notamment par le recours aux «mises en abyme». A plusieurs reprises, on aperçoit un groupe de figurants semblables à des spectateurs qui applaudissent la scène se déroulant sous leurs yeux. A propos du film, R. Ruiz évoquait une "pièce de théâtre montée». Outre la première scène de confrontation entre Lady Anne et Richard, la scène 7 de l'acte III, qui conduit au couronnement de Richard par le maire et les citoyens, offre un autre exemple de «mise en abyme»: les toiles peintes servent de décor à l'arrière-plan pour le duo théâtral de Richard et de Buckingham. Cette mise en scène à la chandelle glisse bien vite vers un théâtre d'ombres car progressivement on ne perçoit plus des deux protagonistes que leurs ombres portées sur des toiles de fond. La 
mise en scène cinématographique illustrerait ici fort à propos la polysémie du mot «shadow», à la fois ombre et acteur. Si pour R. Ruiz, «le théâtre est avant tout un lieu où se rend visible le mystère de l'Incarnation" " ${ }^{17}$, on assisterait paradoxalement dans ce film à un processus de désincarnation de certains personnages : ainsi lors du banquet en plein air, la nuit succède asse $z$ brusquement au soleil couchant tandis que les visages et les formes s'estompent; la scène se poursuit néanmoins dans l'obscurité avec des personnages fantômes dont seules les voix nous parviennent encore. Ces spectres qui apparaissent et disparaissent à la lueur des bougies créent un univers labile aux frontières imprécises. Le film est parcouru de silhoucttes désincarnées, gardes en armes dont on ne sait ce qu'ils gardent ni ce qu'ils attendent et qui participeraient de cet art des ombres, d'une certaine légèreté ontologique constitutive de l'univers ruizien et plus généralement du cinéma.

On note la prédilection du réalisateur pour les écrans qui dissimulent ou déforment le réel : les voiles mauves du deuil, les voilages et tentures derrière lesquels se profilent les personnages, les toiles de fond peintes sur lesquelles se reflètent leurs ombres, les fumées et fumigènes quasi-constants dans les scènes de plein air et d'intérieur, sont autant d'obstacles à une saisie immédiate du monde. Je m'attarderai sur un plan exemplairc montrant ces jeux de détournement et de convergence des regards : R. Ruiz réussit un tour de force en installant sa caméra derrière le voile transparent placé devant le visage de Lady Anne. Tout se passe alors comme si l'œil du spectateur se confondait avec la caméra, elle-même assimilée à l'œil de Lady Anne qui regarde Richard assis à la table. Les multiples reflets de certains personnages dans les miroirs ou les verres de lunettes fourniraient d'autres exemples de ces jeux visuels dont il s'est fait une spécialité.

Maître de l'artifice, R. Ruiz inclut dans son cinéma des citations, des références cinématographiques mais aussi picturales. Comme il l'admet luimême : «c'est un film entièrement parodique : ce sont des parodics qui se lient les unes aux autres, ce sont des jeux de références pour moi» ${ }^{18}$. C'est pourquoi il emprunte volontiers à ses prédécesseurs et ne s'en cache pas. Orson Welles est sans doute la référence la plus évidente. Les premières images du film - ciel tourmenté et eaux bouillonnantes - rappelleraient le début du Macbeth d'O. Welles, tandis que les silhouettes noires des gardes armés filmés à contre-jour feraient écho à la lente progression solennelle du cortège funèbre au début d'Othello. Le jeu sur la disproportion, l'exagération, la multiplicité des points de vue,.les gros plans, l'utilisation de la profondeur de champ, sont aussi des procédés wellesiens reconnus. Certaines images de Richard III, construites sur des contrastes de lumière, des éclairages rasants ou lunaires - en particulier la scène d'apparition des deux meurtriers renverraient quant à elles au cinéma expressionniste des années trente. R. Ruiz. joue avec la forme, les formes, cinématographiques mais aussi picturales, 
comme le prouvent les nombreuses allusions à la peinture.

Le figurant barbu anonyme, interlocuteur muet de Richard dans la taverne, semble inspiré de certains personnages de Bruegel dans La parabole des aveugles, La danse des paysans ou encore Noces villageoises. Dans un plan tourné à l'intérieur de la grotte, la disposition des personnages rappelle étrangement La ronde de nuit de Rembrandt. L'œuf énorme qui trône au milicu de la grotte évoque certains tableaux de Magritte. Il n'est certainement pas anodin qu'à plusieurs reprises, dans la taverne et dans la grotte, des lableaux, accrochés au mur ou disposés sur le sol, soient insérés dans l'image. R. Ruiz ne cache pas son goût pour la composition picturale de certains plans et son attirance pour la peinture. Dans d'autres films - Généalogies d'un crime. L'hypothèse du tableau volé - il va jusqu'à recréer avec les comédicns des tableaux vivants. Ce jeu avec les citations serait l'indice de l'éparpillement, de la disparité qui marque le film, la négation d'une vision unifiće et univoque du réel, au profit de fragments mis bout à bout. «La rupture de styles est ma spécialité», avoue le cinéaste ${ }^{19}$.

Aussi originale et déformante qu'elle soit, l'adaptation ruizienne de Richard III rend pleinement compte d'un univers shakespearien déformé voire disproportionné, «out of joint» pour reprendre l'expression d'Hamlet. R. Ruiz transpose à l'ćcran, avec des moyens cinématographiques, les multiples "shadows", simulacres, fantômes, ombres, apparences sans substance qui hantent le théâtre de Shakespeare. En outre, par le jeu des déformations qu'il met en ceuvre, R. Ruiz montre l'impossibilité du «réalisme ontologique» cher au critique et théoricien André Bazin ${ }^{20}$. Dans un entretien avec F. Revault d'Allonnes, le cinéaste déclare :

Le cinéma a presque toujours «trompé» la réalité, il s'est quasiment toujours démarqué du réel quotidien. En toutes choses, ne serait-ce que par le montage d'abord, qui triche avec la durée, ou par le faux-raccord, les variations de lumière d'un plan à l'autre, les changements de cadres et d'axes, d'objectifs, qui trichent avec l'espace. Chaque fois qu'on déplace le point de vue d'une caméra, on déplace celui du spectateur. Ces évidences cinématographiques n'ont rien à voir avec le réel. ${ }^{21}$

L'image cinématographique ment ; mais le réel lui-même ment. Dans le film de R. Ruiz, Les trois couronnes, un personnage déclare : «Le monde est un mensonge» et un peu plus loin «Le monde est à deux dimensions», deux 
adages ruiziens qui réactualisent et transposent au cinéma le lcitmotiv shakespearien, «All the world's a stage».

Laetitia COUSSEMENT

Université Paris III - Sorbonne Nouvelle

\section{NOTE S}

${ }^{1}$ Il apparaît dans le film Palombella Rossa de Nani Moretti en 1989.

2 L'expression est de Fabrice Revault d'Allonnes dans l'ouvrage écrit en collaboration avec Christine Buci-Glucksmann, Raoul Ruiz, Paris, Dis Voir, 1987 , p. 44.

${ }^{3}$ Interview de Raoul Ruiz dans Télérama n²419, 22 mai 1996.

${ }^{4}$ Par souci de cohérence avec le film de R. Ruiz, la pièce sera citée dans la traduction de J.-M. Déprats, Richard III, Paris, Gallimard, 1995.

${ }^{5}$ Ce rôle était tenu par Charles Tissot dans la mise en scène de G. Lavaudant.

${ }^{6}$ R. Ruiz dans Le Journal des Cahiers du Cinéma, n³75, septembre 1985. p. I

i Voir l'introduction d'Antony Hammond à l'édition Arden de la pièce, Londres et New York, Routledge, 1981, p. 102-3.

8 Voir OED à «shadow», 6b: «also to an actor or a play in contrast with the reality represented». OED relève cet emploi dans Euphues de John Lyly.

9 A. Hammond, op. cit, p. 114.

10 R. Ruiz, «D'une institution à l'autre: entretien avec Raoul Ruiz», Cahiers du Cinéma, n²87, 1978.

11 Eisenstcin est cité par Pascal Bonitzer dans l'article «Métamorphoses», Cahiers du Cinéma, n³45, numéro spécial R. Ruiz, mars 1983.

${ }^{12}$ Serge Toubiana, «Le Cas Ruiz», Cahiers du Cinéma, n³45, mars 1983.

13 R. Ruiz, "Entretien», Cahiers du Cinéma, n³45, mars 1983, p. 76.

14 Alain Bergala, «Le vrai, le faux, le factice», Cahiers du Cinéma, n³51. septembre1983, p. 7.

${ }_{15}$ Pascal Bonitzer, «Richard III, de Raoul Ruiz», Cahiers du Cinéma, n³85, juin 1986 , p. 38.

16 Ibid., p. 38.

${ }^{17}$ R. Ruiz cité par Christine Buci-Glusksmann, Raoul Ruiz, Paris, Dis Voir, 1987 , p. 18.

18 R. Ruiz, «Entretien de R. Ruiz par Bernadette Vignac», Archipel Lavaudant, Paris, Christian Bourgois, 1997, p. 215.

${ }^{19}$ Ibid., p. 215. 
${ }^{20}$ On se reportera à l'article d'André Bazin, «Ontologie de l'image photographique», Qu'est-ce que le cinéma?, vol 1, Paris, Éditions du Cerf, 1975.

21 Christine Buci-Glucksmann et Fabrice Revault d'Allonnes, «Entretien avec Raoul Ruiz», Raoul Ruiz, Paris, Dis Voir, 1987, p. 107. 